\title{
Multi-wavelength observations of the narrow-line Seyfert 1 galaxy RX J2314.9+2243
}

S. Komossa ${ }^{1}$, I. Myserlis ${ }^{1}$, L. Fuhrmann ${ }^{1}$, D. Xu ${ }^{2}$, D. Grupe $^{3}$, Z. Fan $^{2}$, S. Yao $^{2}$, E. Angelakis ${ }^{1}$, V. Karamanavis ${ }^{1}$, J. A. Zensus ${ }^{1}$ and W. Yuan ${ }^{2}$

${ }^{1}$ Max-Planck-Institut für Radioastronomie, Auf dem Hügel 69, 53121 Bonn, Germany email: skomossa@mpifr.de

${ }^{2}$ National Astronomical Observatories, Chinese Academy of Sciences, Beijing, 100012, China

${ }^{3}$ Space Science Center, Morehead State University, 235 Martindale Dr., Morehead, KY 40351, USA

\begin{abstract}
Narrow-line Seyfert 1 (NLS1) galaxies are a sub-class of active galactic nuclei (AGN) with relatively low-mass black holes, accreting near the Eddington rate. A small fraction of them is radio-loud and harbors relativistic jets. As a class, these provide us with new insights into the cause(s) of radio-loudness, the blazar phenomenon at low black hole masses, and the operation of radio-mode feedback. The NLS1 galaxy RXJ2314.9+2243 is remarkable for its multi-wavelength properties. We present new radio observations taken at Effelsberg, and a summary of the recent results from our multi-wavelength study. RXJ2314.9+2243 is radio-loud, luminous in the infrared, has a flat X-ray spectrum and peculiar UV spectrum, and hosts an exceptionally broad and blueshifted [OIII] $\lambda 5007$ emission line, indicating the presence of a strong outflow. RXJ2314.9+2243 likely represents an extreme case of AGN induced feedback in the local universe.
\end{abstract}

Keywords. Black holes, quasars, jets

\section{Radio-loud NLS1 galaxies}

NLS1 galaxies exhibit many extreme properties among AGN, including, on average, super-strong FeII emission, rapid X-ray variability, near-Eddington accretion rates, and rapidly growing low-mass black holes (e.g., Grupe 2004, Xu et al. 2012, review by Komossa 2008). Their radio properties have only been studied systematically in recent years, revealing a number of surprises: as a class, they are less frequently radio-loud than broadline Seyfert 1 galaxies (Komossa et al. 2006), but some of them are beamed (Yuan et al. 2008, Angelakis et al. 2015), $\gamma$-ray detected (Abdo et al. 2009, Foschini et al. 2011), and appear like classical blazars, and at least one of them is puzzling due to its exceptional host galaxy morphology (Zhou et al. 2007).

\section{Results on RX J2314.9+2243}

RX J2314.9+2243 $\left(\alpha_{2000}=23^{\mathrm{h}} 14^{\mathrm{m}} 55.7^{\mathrm{s}} ; \delta_{2000}=+22^{\mathrm{o}} 43^{\prime} 25^{\prime \prime}\right)$ is a radio-loud NLS1 galaxy (Komossa et al. 2006) at redshift $z=0.169$. In order to understand its nature, we have obtained multi-wavelength observations of RX J2314.9+2243 with Swift in the optical, UV and X-rays, with the Xinglong telescopes in the optical (monitoring and spectroscopy), and with the Effelsberg $100 \mathrm{~m}$ telescope in the radio band at multiple frequencies and epochs (see Komossa et al. 2015 for details), including new Effelsberg radio data taken in October 2014. 
Table 1. Radio measurements of RXJ2314.9+2243 performed with the Effelsberg $100 \mathrm{~m}$ telescope. Not all frequencies $\nu$ were observed at all dates. RX J2314.9+2243 was also detected during the NVSS at $1.4 \mathrm{GHz}$ with a flux density of $19 \pm 1 \mathrm{mJy}$.

\begin{tabular}{lccccccc}
\hline \hline$\nu[\mathrm{GHz}]$ & flux density & {$[\mathrm{mJy}]$} & & & & & \\
& 2013 & Feb 03 & 2013 Feb 09 & 2013 July & 2013 July 23 & 2014 Oct 18 & 2014 Oct 31 \\
\hline 2.64 & - & $14 \pm 3$ & $12 \pm 2$ & - & - & - \\
4.85 & $7 \pm 1$ & $7 \pm 1$ & $9 \pm 2$ & $7 \pm 2$ & $10 \pm 2$ & $8 \pm 2$ \\
8.35 & $5 \pm 1$ & $5 \pm 1$ & $5 \pm 1$ & $5 \pm 1$ & $6 \pm 1$ & $5 \pm 1$ \\
10.45 & - & - & $<17$ & - & $<56$ & $<56$ \\
14.60 & - & - & - & - & $<27$ & $<28$ \\
43.00 & - & - & $<56$ & - & $<158$ & $<96$ \\
\hline
\end{tabular}

The spectral energy distribution (SED) of RX J2314.9+2243 shows a broad hump extending between the IR and FUV, a steep downturn in the UV towards shorter wavelengths, a steep radio spectrum and flat X-ray spectrum. Its steep UV spectrum $\left(\alpha_{\mathrm{UV}}=1.6\right)$ is likely intrinsic, since we do not find evidence for strong reddening in the optical spectrum. Its IR to FUV SED is consistent with a scenario, in which synchrotron emission from a jet dominates the broad-band emission, even though an absorption scenario cannot yet be fully excluded.

So far, no variability in any waveband was detected, with the exception of the X-ray band. In the radio regime, its spectrum is steep $\left(\alpha_{\mathrm{R}}=-0.76\right)$, more reminiscent of CSS (compact steep-spectrum sources) than of blazars. Yet, its marginal $\gamma$-ray detection with Fermi (L. Foschini, priv. com.; Berton et al. 2015, in prep.), if confirmed, likely hints at flaring due to a relativistic jet. The observed radio emission likely represents emission from a quiescent jet component, and, indeed, the similar radio fluxes observed with NVSS and at a much later epoch with Effelsberg suggest, that RX J2314.9+2243 was not in a flaring state during the latter observations. New radio observations were carried out in October 2014 (Tab. 1), and the radio emission remains constant within the errors.

The high blueshift of its very broad [OIII] component, $1260 \mathrm{~km} / \mathrm{s}$, is consistent with a face-on view, with the jet (and outflow) pointing towards us. RXJ2314.9+2243 likely represents an extreme case of AGN induced feedback in the local universe.

\section{Acknowledgements}

This work was supported by grant NSFC 11273027. I.M. and V.K. are funded by the International Max Planck Research School (IMPRS) for Astronomy and Astrophysics at the Universities of Bonn and Cologne.

\section{References}

Abdo, A. A., et al. 2009, ApJ, 699, 976

Angelakis, E., Fuhrmann, L., Marchili, N., et al. 2015, A\&SA, 575, A55

Foschini, L., et al. 2011, MNRAS, 413, 1671

Grupe, D. 2004, AJ, 127, 1799

Komossa, S., et al. 2006, AJ, 132, 531

Komossa, S. 2008, Revista Mexicana de Astronomía y Astrofísica Conference Series, 32, 86

Komossa, S., et al. 2015, A\&A, 574, 121

$\mathrm{Xu}$, D., et al. 2012, $A J, 143,83$

Yuan, W., et al. 2008, ApJ, 685, 801

Zhou, H., et al. 2007, ApJ, 658, L13 